\title{
Zu wenig oder zu viel? Warum Dankbarkeit im Rahmen der Gabe-Theorie betrachtet werden sollte
}

\author{
Kommentar zu Thomas Nisters
}

\author{
Hilge Landweer
}

Angenommen: 4. März 2021 / Online publiziert: 26. April 2021

(C) Der/die Autor(en) 2021

Zusammenfassung In Schnitzlers Erzählung „Wohltaten still und rein gegeben“ geht es zentral um das Betteln. Die Hauptfigur erhält eine größere Geldgabe, will aber die Dankesschuld nicht übernehmen, die damit verbunden ist. Der Empfänger der Gabe glaubt, durch das Akzeptieren der Dankesschuld den Status eines Bettlers zugewiesen zu bekommen. Der Beitrag interpretiert Schnitzlers Erzählung neu, und zwar stärker gabentheoretisch. Dies erlaubt eine andere Perspektive als die von Nisters auf die Asymmetrien, die durch Wohltaten entstehen, und wirft die Frage auf, in welchen Fällen sie ausgeglichen werden sollten, falls sie überhaupt ausgeglichen werden können.

Beim Betteln gibt es auf beiden Seiten Unsicherheiten über die Bewertung der Größe der Gabe; in der Erzählung kippt die Bewertung von ,zu groß“ in ,zu wenig“. Allgemein ist beim Geben, so die These, ,zu viel“ ebenso problematisch wie ,zu wenig": Im ersten Fall verpflichtet der Gebende den Empfänger in unstatthafter Weise, nämlich so, dass er sich nicht revanchieren kann und sich dadurch erniedrigt fühlt. Im zweiten kommt der Geber einer unausgesprochenen Verpflichtung nicht nach, dem Anderen vom eigenen Wohlstand in angemessener Weise abzugeben und demütigt den Empfänger durch die Geringfügigkeit. Beim Ausgleich von Gabe und Gegengabe, auch durch Dankbarkeit, geht es nicht um exakte Symmetrie, sondern um Angemessenheit an die Situation.

Eine Dankesschuld, zu der man sich gegen den eigenen Willen und damit zu Unrecht verpflichtet fühlt, kann als dunkle Seite der Dankbarkeit verstanden werden. Eine solche Schuld verwandelt das Gefühl von Dankbarkeit in Ärger.

Schlüsselwörter Dankbarkeit · Gabentheorie · Dankesschuld · Schnitzler: „Wohltaten still und rein gegeben“"

H. Landweer $(\triangle)$ Institut für Philosophie, FU Berlin, Habelschwerdter Allee 30, 14195 Berlin, Deutschland E-Mail: landweer@zedat.fu-berlin.de 
Abstract The subject of Schnitzler's novella „Wohltaten still und reingegeben“ is begging. The main character receives a large donation but does not want to assume the debt of gratitude associated with it. He believes he would be ascribed the status of a beggar if he accepted the debt. The article interprets Schnitzler's story in a new way, relying heavily on the theory of the gift. This allows a different perspective from Nisters' with regard to the asymmetries which occur in cases of benefactions and raises questions concerning how they should be balanced, if they can be balanced at all.

Begging gives rise to uncertainties about the evaluation of the extent of the gift. In the novella, the assessment tilts from "too much" to "too little." In giving, "too much" is generally as problematic as "too little." In the former case, the donor imposes an inadmissible obligation on the beneficiary because the latter cannot return the favor and thereby feels humiliated. In the latter case, the donor does not adequately fulfill a tacit commitment to cede goods or funds to the beneficiary and degrades him with his pettiness. The balance of gift and response, including when the response appears in the form of gratitude, is not achieved through perfect symmetry but is a matter of what is appropriate to the situation.

A debt of gratitude to which one feels committed against one's own will, and thereby unjustly, can be understood as a dark side of gratitude. Such a debt transforms gratitude into anger.

Keywords Gratitude · Theory of the gift · Debt of gratitude · Schnitzler: "Wohltaten still und rein gegeben"

\section{Um welche Asymmetrie geht es bei Undankbarkeit?}

Nisters spricht von ,gratitude-anger“, zusammengezogen zu ,granger“. Ich verstehe das als Ärger über die Zumutung, dankbar sein zu sollen. Undankbarer ,granger“, so Nisters, ist der Dankbarkeit nicht entgegengesetzt, sondern ihr dunkler Bruder. Das scheint mir ein wichtiger Punkt zu sein. Nisters sagt aber nichts zu den Entstehungsbedingungen dieses Gefühls. Eine deutlicher gabentheoretische Sicht könnte diesen Punkt erhellen.

„Granger“ und „gratitude“ weisen nach Nisters drei Gemeinsamkeiten auf: Erstens gehen beide davon aus, dass eine Wohltat eine Asymmetrie hervorbringt, die, zweitens, von beiden nicht toleriert wird und die beide, drittens, auszugleichen versuchen. Im Falle der Dankbarkeit werde der Ausgleich dadurch hergestellt, dass der Empfänger sich selbst durch das Gefühl aufwerte, im Falle des ,granger“" werde der Wohltäter abgewertet und seine Position untergraben.

Die Annahme, dass durch eine Wohltat eine Asymmetrie hergestellt werde, die ausgeglichen werden müsse, hat auf den ersten Blick Anklänge an die Gabentheorie. Nach der Theorie von Marcel Mauss gehören zum Gabentausch drei Elemente (Mauss 1989 [1925]): das Geben, das Empfangen und das Erwidern der Gabe. Sie verpflichtet den Empfänger, weil sie freiwillig erfolgt und bereitwillig empfangen wird, und hat die Funktion, die Beziehung von zwei Gruppen zu festigen und die Konfliktgefahr abzuwehren. Die drei ,Stationen“ beim Gabentausch werden unter 
symbolisch gleichen Partnern in einer endlosen Kette ständig in einer gewissen, zeitlich nicht genau festgelegten Abfolge wiederholt und stabilisieren so die Beziehung. Bei Marcel Mauss selbst spielt die Dankbarkeit keine zentrale Rolle, aber neuere Diskussionen gehen davon aus, dass sie entweder selbst als Gegengabe betrachtet werden kann (Derrida 1993) oder aber als diejenige Haltung, die als angemessen gilt, um die Zeit bis zur Gegengabe zu überbrücken und die Erinnerung an die empfangene Gabe wachzuhalten (nicht explizit gabentheoretisch: Bollnow 1954).

Um welche Art von Asymmetrie und entsprechend um welchen Ausgleich geht es bei Nisters? Er stützt sich zur Veranschaulichung des ,granger“ auf Arthur Schnitzlers Erzählung „Wohltaten still und rein gegeben“, ${ }^{1}$ einen mehrschichtigen Text, der nicht nur den Umschlag von Dankbarkeit zu einem aggressiven Vergeltungsakt anspricht. Die Erzählung kann, etwas anders als Nisters es tut, gabentheoretisch gedeutet werden. Dies erlaubt auch eine andere Perspektive auf die Asymmetrien, die durch Wohltaten entstehen, und auf die Frage, in welchen Fällen - und vor allem wie - sie ausgeglichen werden sollten, und ob sie überhaupt ausgeglichen werden können.

Die Wohltat in Schnitzlers Erzählung ist, das sei vorausgeschickt, gerade keine, die eine Beziehung herzustellen oder zu festigen versucht. Vielmehr handelt es sich dabei um eine Geldgabe, die den Empfänger in die Position eines Bettlers bringt. Bei einer Spende für einen Bettler handelt es sich, gabentheoretisch betrachtet, von vornherein um eine einseitige Gabe, nicht um eine, die erwidert werden muss (vgl. Hénaff 2009). Aber so eindeutig liegen die Dinge nicht in dieser Erzählung, und genau das macht ihre Faszination und die (auch gaben-) theoretische Herausforderung aus, die von ihr ausgeht. Deshalb verspricht eine Re-Lektüre lohnend zu sein.

\section{Re-Lektüre von Schnitzlers Erzählung}

In Schnitzlers Erzählung setzt sich die Hauptfigur, Franz, von Beginn an mit dem Betteln auseinander; keinesfalls möchte er als Bettler angesehen werden. Er beobachtet jemanden, der bei den vor einem hell erleuchteten Saal vorfahrenden Wagen die Türen öffnet und dafür Trinkgeld erhält. ,In Franz regte sich der Neid. Wenn er sich doch auch zu dergleichen entschließen könnte. Aber das war ja schon Bettelei. Und er war Student..." Franz ist vor Hunger in einer Verfassung, in der er seine Gefühle hemmungslos auf andere projiziert: „Ein junger Mann und eine Dame gingen an ihm vorüber; sie aßen gebratene Kastanien und lachten, als ob sie das sehr komisch fänden, daß sie naschen durften, während andere hungerten." Die latente Aggression wird immer deutlicher: „Am liebsten hätte er ihnen die warmen duftenden Dinger einfach aus der Hand gerissen und verschlungen, und er spürte, daß ihm zu einer solchen Handlung eigentlich nichts fehlte als der Mut." Hier keimen Wut und Zorn auf, bevor irgendeine Wohltat erfolgt ist. Im inneren Monolog nennt Franz sich einen ,feigen Hungerleider“ und überlegt, dass er doch besser zu Hause geblieben und Handwerker oder Landwirt geworden wäre. Es geht also auch um

\footnotetext{
1 http://www.zeno.org/Literatur/M/Schnitzler,+Arthur/Erzählungen/Wohltaten+Still+und+Rein+gegeben.
} Alle Zitate im Folgenden beziehen sich auf diese nicht in Seiten untergliederte Ausgabe. 
eine Identitätsfrage: „Daheim hätt' er schon irgendwie ehrlich sein Brot verdient, ohne sich demütigen zu müssen wie hier. Was war aus ihm geworden!“‘

Seine Gefühle schwanken zwischen Aggressionsgefühlen (z.B. Neid; aber eben auch verschiedene Formen von ,,anger“, vgl. Landweer 2020a), Sich-gedemütigt fühlen und Scham. Die letzten beiden Gefühle können als moralische Gefühle bezeichnet werden in dem Sinne, dass sie sich auf Unrecht beziehen können: Bei Scham hat man selbst eine Norm übertreten, die man mindestens ambivalent anerkennt, im Gefühl des Gedemütigt-werdens fühlt man sich ohnmächtig und ausweglos in eine Enge gedrängt angesichts eines Unrechts, das einem von jemand anderem (oder auch ohne klare Zurechenbarkeit auf jemand Bestimmten) zugefügt wird (Landweer 2016). Beide Gefühle werden in der Philosophie und im öffentlichen Diskurs leicht verwechselt. Betroffene schwanken oft zwischen diesen Emotionen, weil sie in der Zurechnung der Verantwortung für ihre Lage unsicher sind: Hielten sie eindeutig sich selbst als zuständig für die Normüberschreitung, so würden sie sich schämen. Franz schämt sich aber nicht; seine Scham kommt erst später und zu anderen Anlässen ins Spiel. Er findet nicht, dass er verantwortlich für seine Armut ist.

Dennoch nimmt er eine zwiespältige Haltung zu sich selbst ein, als ein junger Mann im Pelz ,plötzlich“ vor ihm stehen bleibt und mit ,weißbehandschuhter Hand“ seine Geldbörse hervorzieht: „Gegen seinen Willen, ja mit einem dumpfen Staunen über sich selbst, hielt Franz die Augen auf den Herrn fast flehentlich gerichtet und die Rechte wie zum Empfang ausgestreckt.“ Franz ist offenbar über sich selbst überrascht, sich - als Antwort auf das Verhalten des anderen - in einer Bettelgeste vorzufinden. Nun sucht der Herr in seiner Börse, findet offenbar keine kleineren Münzen und gibt ihm ein Zehnkronenstück. An Franz' reflexhafter Reaktion wird deutlich, dass die Gabe für eine derartige Situation ungewöhnlich hoch ist: „Franz riß unwillkürlich Mund und Nase auf. Ein ungeheures Entzücken erfüllte ihn.“ Der Höhe der erhaltenen Gabe entsprechend fällt Franzens folgende Handlung aus: „Mit Inbrunst faßte er die Hand des jungen Mannes - drückte sie und preßte sie endlich an die Lippen“, eine Geste, auf die wiederum sein Gegenüber mit Überraschung reagiert: „Dieser wich, wie erstaunt, zurück, schien etwas fragen zu wollen, rasch aber besann er sich, [...] und verschwand in der Halle.“ Dieses Verhalten deutet auf Verlegenheit hin, Unsicherheit, Zögern, die ihren Grund darin zu haben scheinen, dass die gegebene Summe zu hoch, aber auch Franz' spontane Dankesgeste aus der Sicht des anderen ungewöhnlich oder nicht angemessen war.

Diese Szene hat ein Pendant in einer Situation, die weiter hinten in der Erzählung geschildert wird. Franz sitzt inzwischen, versunken in sehnsüchtige Gedanken, in einer Kneipe. Ein „,blasses“ Mädchen will ihm, ohne ihn direkt anzusprechen, ein Blumensträußchen verkaufen. Schnitzler schreibt: „In seiner Verlegenheit griff er wie mechanisch nach dem Sträußchen [...]“ Aus dieser Verlegenheit wird sofort eine Verlegenheit des Mädchens: „Während er nach einem Zwanzighellerstück suchte, nahm das Mädchen die Blumen vom Tisch auf und steckte sie ihm ins Knopfloch, immer schweigsam, ohne die Miene zu verziehen, als dächte sie an etwas ganz anderes." Auch das Mädchen möchte offenbar nicht mit dieser dem Betteln nicht ganz unähnlichen Situation identifiziert werden. Als Franz dann schließlich ein Geldstück hervorgeholt hat, sieht er, dass es sich um eine Krone handelt, also um das Fünffa- 
che dessen, was er beabsichtigt hatte: „[...], aber er schämte sich, weiterzusuchen“ und gibt ihr die Münze. Sie antwortet darauf mit dem Satz „Küss’ die Hand, gnädiger Herr", eine verbale Reminiszenz an Franz' tatsächlichen Handkuss vorher. „[D]er Klang dieser Worte durchbebte ihn“, er fühlt sich gleich besser und glaubt, die umgebenden Menschen würden ihn nach dieser Äußerung des Mädchens mit Hochschätzung ansehen.

Zwischen diesen beiden Szenen, die den Empfang einer unerwartet großen Gabe beschreiben, distanziert sich Franz an zwei Stellen in inneren Monologen von seiner Reaktion auf den Mann, der ihm die zehn Kronen geschenkt hatte:

Mit Unbehagen dachte er an den Moment, in dem er dem jungen Herrn flehentlich die Hand entgegengestreckt hatte, und noch viel ärgerlicher an den Augenblick, der gleich darauf gefolgt war... Er hatte nicht übel Lust, wieder zum Sophiensaal zurückzukehren, um dort seinen Wohltäter beim Ausgang abzupassen und ihm zu erklären, daß er keineswegs ein Bettler sei.

Franz geht es also darum, diese Zuschreibung zurückzuweisen, die in der Erzählung ausschließlich durch ihn selbst geschieht. Dass der „Augenblick“ des Handkusses an dieser Stelle unbenannt bleibt und die Aussparung durch drei Auslassungspunkte unterstrichen wird, lese ich als einen Hinweis auf Scham. Scham ist ein Gefühl, das, wenn es intensiv ist, nicht ausgesprochen werden darf, um nicht noch unerträglicher zu werden - es wird verschwiegen und versteckt; jede Benennung wäre eine Wiederholung, ein Heraufbeschwören der beschämenden Situation. Während es lediglich unbehaglich ist, an das Entgegenstrecken der Hand zu denken, ist der Gedanke an den Handkuss sofort wegzuschieben, er ist zu beschämend - und deshalb ärgerlich. Franz schämt sich also nicht für seine Armut, sondern für den leidenschaftlichen (,mit Inbrunst“) Handkuss. Gegen welche Norm hat er verstoßen, dass er von Scham ergriffen wird? Franz hat zwei Anlässe für Scham: Erstens scheint ihm seine Dankesgeste nachträglich als unangemessen groß und übertrieben, und zweitens könnte er der Auffassung sein, dass er sich mit dieser Geste der Dankbarkeit gewissermaßen daran beteiligt hat, als Bettler wahrgenommen werden zu können. Warum sonst äußert er unmittelbar nach der Erinnerung an den Handkuss das starke Bedürfnis, seinem „Wohltäter“ zu erklären, dass er „keineswegs“ ein Bettler sei? Den Wohltäter wird das wenig interessieren, Franz selbst aber betrifft es unmittelbar und umfassend. Geradezu obsessiv ist er in seiner Fantasie mit dem jungen Mann befasst: „Der tanzte wohl in diesem Augenblick“, heißt es in einer späteren Situation, der zweiten Distanzierung,

[...] und hatte das stolze Gefühl, einen armen Teufel glücklich gemacht, zu ewigem Dank verpflichtet zu haben. Franz biß sich in die Lippen ... Ja, wenn es zehnmal soviel gewesen wäre - oder hundertmal!... Ja, dann hätte er ihm wohl die Hand küssen dürfen. Dann hätte er seine Existenz von Grund auf ändern, ein neues Leben beginnen, ein Mensch... ein Mensch wie andere Menschen werden können!... Aber dieses eine Goldstück!... Es war gerade genug, um ihn seine Armut noch bitterer empfinden zu lassen und ihn tiefer zu erniedrigen als je zuvor... Die Erinnerung trieb ihm die Schamröte in die Wangen... (Auslassungspunkte im Text) 
Dem - von Franz imaginierten - Stolz des Gebers wird hier die Scham des Empfängers und sein Gefühl, erniedrigt und gedemütigt worden zu sein, entgegengesetzt. Anfangs ist Franz, wie gezeigt, über die Größe der Gabe erstaunt, interpretiert sie aber jetzt als zu gering. In der Projektion, wonach der Geber angeblich boshafterweise einen ,armen Teufel [...] zu ewigem Dank“"verpflichten wollte, wird indirekt deutlich, dass eine solche Gabe üblicherweise als ,zu groß“ oder zumindest als sehr großzügig betrachtet wird (denn sonst könnte Franz dem freigiebigen jungen Mann wohl kaum die Überzeugung zuschreiben, ihn zu ,ewigem Dank“ verpflichtet zu haben), anstatt als zu gering, wie er anschließend behauptet. Es hätte zehnoder gar hundertmal so viel sein sollen, um aus seiner jetzigen Sicht den Handkuss zu rechtfertigen. Aufschlussreich ist auch die Formulierung, dann hätte er ihm die Hand küssen dürfen, so als gäbe es eine Regel, ab welchem Betrag ein Handkuss angemessen gewesen wäre und als bedürfe es einer Erlaubnis für diese Geste. Diese Erlaubnis gibt Franz sich jetzt nicht mehr, er bestraft vielmehr sich selbst und seine allzu dankesbereiten Lippen, indem er darauf beißt. Er empfindet durch die Gabe seine Armut inzwischen bitterer, ja, er fühlt sich durch sie tiefer erniedrigt ,als je zuvor".

\section{Die Bettelgabe: stets zu wenig oder stets zu viel?}

Diese Dramatik wie auch die der gesamten Erzählung entsteht daraus, so meine Interpretation, dass es beim Betteln auf beiden Seiten Unsicherheiten über die Bewertung der Größe der Gabe gibt: Der Geber weiß, dass es in gewisser Weise immer zu wenig ist, was er gibt, und der Empfänger empfindet es genauso, denn die gesellschaftliche Position beider verändert sich nicht durch die Wohltat. Andererseits wissen die Beteiligten in dieser Erzählung, dass die Gabe hier zu groß ist, weil sie die Konvention kennen, die bestimmt, welcher Spielraum für solche Gaben vorgesehen ist, und empfinden deshalb Unbehagen. Im Verlauf der Erzählung kippt die Bewertung von ,zu groß“ in ,zu wenig“.

Der theoretisch interessante Punkt ist, so meine These, dass „zu viel“ beim Geben ebenso problematisch ist wie ,zu wenig“: Im ersten Fall verpflichtet man den Empfänger in unstatthafter Weise, nämlich so, dass er sich nicht revanchieren kann und sich dadurch erniedrigt fühlt. Im zweiten kommt man einer unausgesprochenen Verpflichtung nicht nach, dem Ärmeren vom eigenen Wohlstand in angemessener Weise abzugeben und demütigt den Empfänger durch die Geringfügigkeit - so als ob er nicht mehr wert wäre (kein Mensch wie andere Menschen ist, wie Franz oben sagt).

Das Problem des Bettelns liegt darin, dass von vornherein eine soziale Asymmetrie besteht, die durch keine individuelle Gabe ausgeglichen werden kann. Deshalb ist eine Gabe an einen Bettler, gabentheoretisch gesehen, von vornherein zu groß und zu klein: zu groß, weil es eine erste Gabe ohne Vorgeschichte ist (,Wohltaten still und rein gegeben“ lautet der Titel der Erzählung) und der Gebende dem Empfänger ,nichts schuldig“ ist, aber auch, insofern letzteren die Gabe, wenn sie nicht gerade erschreckend gering ausfällt, tatsächlich ,zu ewigem Dank“ verpflichtet, da er sie voraussichtlich niemals wird zurückgeben können. Zugleich ist die Gabe aber 
auch immer zu klein, weil sie die Ungleichheit zwischen Geber und Empfänger nicht auszugleichen und den Empfänger sogar in eine noch tiefere Asymmetrie zu stürzen vermag. Letzteres ist paradoxerweise gerade dann der Fall, wenn sie über dem Üblichen liegt, denn je größer sie ist, zu umso mehr Dank verpflichtet sie - ein Dank, den der bedürftige Empfänger nicht mit einer Gegengabe auflösen kann.

Franz fühlt sich durch die Gabe degradiert. Er hatte nicht gebettelt. Ihm geht es um die Zurückweisung des Bettelstatus. Denn mit der „Wohltat still und rein gegeben“ wird ihm auch dieser Status quasi zwangsläufig zugewiesen. Dagegen wehrt er sich. Die ,mächtige“ Ohrfeige am Schluss kompensiert deshalb nicht nur die unangemessen große Dankesgeste, den Handkuss, sondern, wichtiger noch, Franz will damit deutlich machen, dass er kein Bettler ist; er behauptet sich mit der Ohrfeige gegen eine solche Unterstellung. Er weist damit die Gabe nachträglich zurück (und setzt sich selbst damit natürlich ins Unrecht, weil er die Gabe bereits angenommen hat).

Ich verstehe die dunkle Seite der Dankbarkeit so, dass es sich dabei um eine „Dankesschuld“ handelt, zu der man sich gewissermaßen gegen den eigenen Willen und damit zu Unrecht verpflichtet fühlt. Daraus entsteht ein unangenehmes Gefühl, das mit Zorn einhergehen kann. Das Problem kommt auf, wenn die Freiheit, eine Gabe abzulehnen, nicht mehr existiert. Das kann verschiedene Gründe haben. Oft wäre es schlicht ein zu großer Affront, die Gabe zurückzuweisen. Das unangenehme Gefühl stellt sich vor allem bei unangemessenen, d.h. zu großen (ersten) Gaben oder bei Gaben von Personen ein, mit denen man nicht verbunden sein möchte und denen man deshalb ausweicht. Das eine mal steht bei der Abwehr der Dankbarkeit die Gabe, das andere Mal die Person des Gebers im Mittelpunkt, und natürlich ist beides oft vermischt. Das kann zur Distanzierung von der Person des Gebers oder sogar zum Ärger über die zugemutete Dankbarkeit führen. Dieser Ärger entsteht besonders leicht, wenn man sich zur Annahme der Gabe vielleicht gerade durch ihre beeindruckende Größe gewissermaßen hat verführen lassen oder nicht anders konnte, als sie anzunehmen. Bei Franz ist letzteres der Fall: Er konnte in seiner Situation die Gabe nicht ablehnen, er wäre womöglich verhungert. Dabei geht es Schnitzler, so meine Interpretation, zwar auch ein wenig um Dankesschuld, aber stets auf der Folie des Bettelns: Aus Franz' Perspektive ist die Dankesschuld abzuweisen, weil sie ihm fälschlich den Status eines Bettlers zuweist. Das Betteln aber muss gabentheoretisch ganz anders betrachtet werden als der ,normale" Gabentausch zwischen symbolisch Gleichen.

Auch wenn ,granger“ als ein Unrechtsgefühl angesehen werden kann, das tatsächlich auf Ausgleich zielt, so scheint mir Nisters' Annahme, beim Gabentausch bzw. bei Dankbarkeit gehe es vor allem um das Wiederherstellen einer Symmetrie, doch in die falsche Richtung zu gehen. Es geht in der Dankbarkeit nicht primär um Aufwertung oder Abwertung des Gebers bzw. des Empfängers. Ziel ist vielmehr, anders als beim Betteln, die Herstellung bzw. Festigung einer Beziehung. Die Vergleichbarkeit von Gabe und Gegengabe (hier: der Dankbarkeit), die keinen ökonomischen, sondern nur einen symbolischen Wert haben, wird nicht durch irgendeinen Maßstab oder eine Symmetrie hergestellt, sondern durch Angemessenheit. Angemessenheit lässt sich nicht in ein allgemeines Prinzip überführen. Sie ist ein intuitives emotionales Vermögen, das in ihre Bewertung den Status von Geber und Empfänger, die Beziehung und ihre Geschichte sowie vor allem: die Situation 
mit einbezieht (Landweer 2020b). Nisters kommt einer gabentheoretischen Deutung nahe, aber übersieht die vorgängige Status-Asymmetrie. Gabentheoretische Erwägungen funktionieren nur unter symbolisch Gleichen. Das ist beim Betteln gerade nicht der Fall.

Nisters scheint sich die Frage zu stellen, ob „granger“ unter bestimmten Umständen moralisch akzeptabel sein kann. Erklärungsbedürftig ist dabei der ,unsägliche Haß“, der in Franz aufgrimmt. Er könnte das Resultat eines Unrechtgefühls sein, das keine Kompensation finden kann. Vor allem deshalb nicht, weil nicht klar ist, wer für Franz' schwierige Situation verantwortlich ist. Deshalb bleibt Franz' Aggression, die den (gar nicht ausgesprochenen) Bettelvorwurf zurückweisen soll, richtungslos bzw. richtet sich stellvertretend gegen den Geber.

Ärger über zugemutete Dankbarkeit ist zwar kein moralisches Gefühl; aber als ein Gefühl, das sich darauf bezieht, dass ein Ausgleich unter den gegebenen Bedingungen nicht möglich ist, könnte man ihn vielleicht als ,vormoralisch“ bezeichnen, als ein Gefühl, das auf ein moralisches Problem hinweisen kann. Das würde gut zur Dankbarkeit passen, die nicht moralisch gefordert werden kann (Emundts 2017), aber doch als supererogatorisch anzusehen ist, weil sie über Pflicht hinausgeht.

Funding Open Access funding enabled and organized by Projekt DEAL.

Open Access Dieser Artikel wird unter der Creative Commons Namensnennung 4.0 International Lizenz veröffentlicht, welche die Nutzung, Vervielfältigung, Bearbeitung, Verbreitung und Wiedergabe in jeglichem Medium und Format erlaubt, sofern Sie den/die ursprünglichen Autor(en) und die Quelle ordnungsgemäß nennen, einen Link zur Creative Commons Lizenz beifügen und angeben, ob Änderungen vorgenommen wurden.

Die in diesem Artikel enthaltenen Bilder und sonstiges Drittmaterial unterliegen ebenfalls der genannten Creative Commons Lizenz, sofern sich aus der Abbildungslegende nichts anderes ergibt. Sofern das betreffende Material nicht unter der genannten Creative Commons Lizenz steht und die betreffende Handlung nicht nach gesetzlichen Vorschriften erlaubt ist, ist für die oben aufgeführten Weiterverwendungen des Materials die Einwilligung des jeweiligen Rechteinhabers einzuholen.

Weitere Details zur Lizenz entnehmen Sie bitte der Lizenzinformation auf http://creativecommons.org/ licenses/by/4.0/deed.de.

\section{Literatur}

Bollnow, Otto Friedrich. 1954. Über die Dankbarkeit. Die Sammlung, 9. Jahrg., 169-177.

Derrida, Jacques. 1993. Falschgeld: Zeit geben I. München: Fink.

Emundts, Dina. 2017. Zur Rolle von Dankbarkeit in Moral und Recht. In: Recht und Emotion II. Sphären der Verletzlichkeit, 99-141. Hrsg. Hilge Landweer, Fabian Bernhardt. Freiburg: Alber.

Hénaff, Marcel. 2009 (Frz. zuerst 2002). Der Preis der Wahrheit. Gabe, Geld und Philosophie. Frankfurt a. M.: Suhrkamp.

Landweer, Hilge. 2016. Ist Sich-gedemütigt-fühlen ein Rechtsgefühl? In: Recht und Emotionen I. Verkannte Zusammenhänge, 103-135. Hrsg. Hilge Landweer, Dirk Koppelberg. Freiburg: Alber.

Landweer, Hilge 2020a. Aggressive Emotions: From Irritation to Hatred, Contempt and Indignation. In: The Routledge Handbook of the Phenomenology of Emotions, 441 - 454. Ed. Thomas Szanto, Hilge Landweer. London/New York.

Landweer, Hilge. 2020b. Warum Normen allein nicht reichen. Sinn für Angemessenheit und Rechtsgefühl in rechtsästhetischer Perspektive. In: Rechtsästhetik in rechtsphilosophischer Absicht, 63 - 84. Hrsg. Eva Schürmann, Levno von Plato. Baden-Baden: Nomos.

Mauss, Marcel. 1989 (Frz. zuerst 1925). Die Gabe. Form und Funktion des Austauschs in archaischen Gesellschaften. Soziologie und Anthropologie 2, 11-144. Frankfurt a. M.: Fischer. 